\title{
UNIFORM BOUNDS FOR ISOPERIMETRIC PROBLEMS
}

\author{
JERROLD SIEGEL AND FRANK WILLIAMS
}

(Communicated by Frederick R. Cohen)

\begin{abstract}
In this paper we generalize our previous joint work with Allan Calder on the width of homotopies by considering an arbitrary finite polyhedral pair $(W, V)$ rather than $(I,\{0,1\})$. We show that given appropriate topological conditions on a Riemannian manifold $M$, with respect to $(W, V)$, there are bounds, $B_{q}(a,(W, V), M)$, such that if $F: K \times W \rightarrow M$ is a map with $\operatorname{Lip}(F \mid(K \times V))<a$, then $F$ can be deformed $\operatorname{rel}(K \times V)$ to $F^{\prime}$ with $\operatorname{Lip}\left(F^{\prime}\right)<B_{q}(a,(W, V), M)+\varepsilon$, where $\varepsilon>0$ is arbitrary and $\operatorname{dim}(K)=q$.
\end{abstract}

\section{INTRODUCTION}

In a sequence of papers ([1], [3], [9], [10], and [11]) Allan Calder and the present authors studied certain families of invariants for Riemannian manifolds with finite fundamental groups. These invariants were based on the concept of the "width" of a homotopy into the manifold. Regarding a homotopy $H: K \times$ $I \rightarrow M$ as a continuous family of paths, the width of $H,|H|$, is the supremum of lengths of these paths. The central result of our earlier work was that for each $n$ there existed a global bound $B_{q}(M)$ such that for all $K$ with $\operatorname{dim}(K)<q$, $H$ can be deformed $\operatorname{rel}(K \times\{0,1\})$ to a homotopy $H^{\prime}$ with $\left|H^{\prime}\right|<B_{q}(M)+\varepsilon$, where $\varepsilon$ may be chosen arbitrarily small.

In this paper we study whether similar results hold when one replaces the pair $(I,\{0,1\})$ with an arbitrary finite polyhedral pair $(W, V)$. We find that the answer is yes, given certain topological conditions determined by the particular manifold and polyhedral pair. The proofs, however, are more involved and highlight the fact that the functional underlying our earlier work was the Lipschitz constant, not the path length. (There is a small literature on related aspects of the Lipschitz constant, cf. [4], [7], and [8].)

We shall consider data consisting of a compact metric polyhedral pair $(W, V)$ and a closed Riemannian manifold $M$. For a map $f: W \rightarrow M$, let $\operatorname{Lip}(f)$ denote the Lipschitz constant of $f$ :

$$
\operatorname{Lip}(f)=\sup _{x \neq y \in W}\left\{\frac{d_{M}(f(x), f(y))}{d(x, y)}\right\} .
$$

Received by the editors December 16, 1988.

1980 Mathematics Subject Classification (1985 Revision). Primary 53B20, 58E99; Secondary $55 \mathrm{P} 99$. 
A form of the isoperimetric question is: Given a map $f: W \rightarrow M$ with $\operatorname{Lip}(f \mid V)<\infty$, what is the infimum of the set of $\operatorname{Lip}\left(f^{\prime}\right)$ where $f^{\prime}$ agrees with $f$ on $V$ ? More generally, we may ask a parametric version of this question: Given a map $F: K \times W \rightarrow M$ with $\operatorname{Lip}(F \mid(K \times V))<\infty$, what is the infimum of the set of $\operatorname{Lip}\left(F^{\prime}\right)$ such that $F^{\prime}$ agrees with $F$ on $K \times V$ ? Here

$$
\operatorname{Lip}(F)=\sup _{x \in K}\left\{\operatorname{Lip}\left(F_{x}\right), \text { where } F_{x}(w)=F(x, w)\right\}
$$

In this paper we prove boundedness theorems for solutions to these variational problems. An example of the results we obtain is

Theorem A. Let $n, q$ be nonnegative integers and $\left(D^{n}, S^{n-1}\right)$ be the unit ball in $\mathbf{R}^{n}$. Let $M$ be a closed Riemannian manifold with $\pi_{n}(M)$ finite. For every $a \geq 0$ there exists $\tilde{b}<\infty$ such that if $F: K \times D^{n} \rightarrow M$ is a map with $\operatorname{Lip}(F \mid$ $\left.\left(K \times S^{n-1}\right)\right) \leq a$ and $\operatorname{dim}(K) \leq q$, then $F$ can be deformed $\operatorname{rel}\left(K \times S^{n-1}\right)$ to $\widetilde{F}$ with $\operatorname{Lip}(\widetilde{\widetilde{F}})<\tilde{b}$.

Thus $B_{q}\left(a,\left(D^{n}, S^{n-1}\right), M\right)=$ infimum of all such $\tilde{b}$ is an invariant of $M$ that provides a bound on the solutions of a class of parametrized isoperimetric problems.

We remark that the requirement that $\pi_{n}(M)$ be finite is, in general, necessary. In fact, we have

Theorem B. $B_{1}\left(1,\left(D^{n}, S^{n-1}\right), S^{n}\right)=\infty$.

The nonparametrized case, $q=0$, is already of interest. We observe

Theorem C. Let $(W, V)$ be a compact polyhedral pair and $M$ be a closed Riemannian manifold. Then for every $a \geq 0$ there exists $\tilde{b}$ such that if $f: W \rightarrow M$ is any map with $\operatorname{Lip}(f \mid V) \leq a$, then $f$ may be deformed, $\operatorname{rel}(V)$, to a map $\tilde{f}$ with $\operatorname{Lip}(\tilde{f})<\tilde{b}$.

The relationship between the results of the present paper and our earlier work can be seen by observing that Theorem A remains true if one replaces the second occurrence of Lip by any other functional dominated by the Lipschitz constant, $k$-dimensional volume, $k$-content, or $k$-dimensional Hausdorff measure. However, one cannot make similar statements about the condition on $K \times S^{n-1}$. The critical point is that the space of Lipschitz maps from $S^{n-1}$ to $M$ with a given bound is compact. This is not the case for the other functionals just mentioned.

For completeness, it is worth remarking that in our earlier work we were considering the pair $\left(D^{1}, S^{0}\right)$. In this case, the space of maps of $S^{0}$ into $M$ is trivially compact.

One would like to be able to compute some of these new invariants as we were able to compute those in our earlier work, but the present state of knowledge of the homotopy of function spaces does not appear sufficient to permit this, even in simple cases. In particular, it would be useful to know for, say, $M=S^{n}$ 
and $(W, V)=\left(D^{2}, S^{1}\right)$, how the double-loop space $\Omega^{2} S^{n}$ acquires its cellular structure in terms of the Lipschitz constant.

$\S 2$.

We begin by formalizing the definitions presented in $\S 1$. Let us fix a compact metric polyhedral pair $(W, V)$ and a closed connected Riemannian manifold $M$.

2.1. Definition. Let $K$ be a finite-dimensional polyhedron and $F: K \times W \rightarrow M$ a map. Set

(a) $L(F)=\inf \{\operatorname{Lip}(\tilde{F}) \mid \widetilde{F} \simeq F \operatorname{rel} K \times V\}$;

(b) $\operatorname{Map}_{q}(a,(W, V), M)=\{F \mid \operatorname{Lip}(F \mid K \times V) \leq a$, and $\operatorname{dim}(K) \leq q\}$; and

(c) $B_{q}(a,(W, V), M)=\sup \left\{L(F) \mid F \in \operatorname{Map}_{q}(a,(W, V), M)\right\}$.

As the following example shows, these quantities will, in general, be infinite.

Proof of Theorem B. $\left(B_{1}\left(1,\left(D^{n}, S^{n-1}\right), S^{n}\right)=\infty\right)$. Let $d$ be a positive integer. Let

$$
f_{d}:\left(I \times S^{n-1},\left(\operatorname{Bd}(I) \times S^{n-1}\right) \cup(I \times\{*\})\right) \rightarrow\left(S^{n}, *\right)
$$

be a map of degree $-d$ such that $L\left(f_{d}\right) \leq 1$. (Recall that this means that for each $t \in I, f_{d}:\{t\} \times S^{n-1} \rightarrow S^{n}$ has Lipschitz constant $\leq 1$.) Let $g_{d}: I \times D^{n} \rightarrow$ $S^{n}$ be any extension of $f_{d}$. Finally, let $g_{d}^{+}$and $g_{d}^{-}$be the restrictions of $g$ to $\{1\} \times D^{n}$ and $\{0\} \times D^{n}$ respectively. $\operatorname{Bd}\left(D^{n} \times I\right)$ is the union of the (closures of the) three $n$-cells $\{1\} \times D^{n},\{0\} \times D^{n}$, and $S^{n-1} \times I$. Since $g_{d} \mid \operatorname{Bd}\left(D^{n} \times I\right) \sim *$, we see that

Hence

$$
\operatorname{deg}\left(f_{d}\right)+\operatorname{deg}\left(g_{d}^{+}\right)+\operatorname{deg}\left(g_{d}^{-}\right)=0 .
$$

$$
\max \left\{\operatorname{deg}\left(g_{d}^{+}\right), \operatorname{deg}\left(g_{d}^{-}\right)\right\} \geq d / 2 .
$$

Since $d$ was arbitrary, $B_{1}\left(1,\left(D^{n}, S^{n-1}\right), S^{n}\right)=\infty$. Q.E.D.

We proceed to our most general result after recalling two relevant facts.

2.2. Lemma. (The Bounded Lifting Lemma [1, Theorem 2.3]).

Let $p: E \rightarrow B$ be a fibration with fiber $F$ and such that

(a) $B$ is compact;

(b) $p$ is locally fiber-homotopy trivial; and

(c) $F$ is the homotopy type of a $C W$-complex of finite type.

Then for each $n$ there is a compact subspace $C_{n} \subseteq E$ such that if $X$ is $n$ dimensional and $f: X \rightarrow E$ is a map, we may deform $f$ to $f^{\prime}$ over $p$ such that $f^{\prime}(X) \subseteq C_{n}$.

2.3. Lemma. Let $M$ be a compact Riemannian manifold. We can choose $U$, a neighborhood of the diagonal in $M \times M$, and $\kappa \geq 0$ such that

(a) if $(x, y) \in U$ there is a unique minimal geodesic, $\lambda(x, y, t)$, in $U$ from $x$ to $y$, and 
(b) there is a finite convex covering $\left\{V_{i}\right\}$ of $M$ such that for all $i, V_{i} \times V_{i} \subseteq$ $U$ and such that for $x, y_{1}, y_{2} \in V_{i}$ and $0 \leq s \leq 1$,

$$
d\left(\lambda\left(x, y_{1}, s\right), \lambda\left(x, y_{2}, s\right)\right) \leq \kappa d\left(\lambda\left(x, y_{1}, 1\right), \lambda\left(x, y_{2}, 1\right)\right) .
$$

(See, for example, [2].)

2.4. Theorem. Let $(W, V)$ be a compact metric polyhedral pair and $M$ a closed connected Riemannian manifold. Suppose that for every map $f: V \rightarrow$ $M$ the space $\operatorname{Fib}(f)=\{\bar{f}: W \rightarrow M \mid \bar{f}$ extends $f\}$ has a finite number of components. Then for all $a \geq 0, q \geq 0$ we have $B_{q}(a,(W, V), M)<\infty$.

Theorem $\mathrm{A}$ is a special case of this theorem, since when $(W, V)=\left(D^{n}, S^{n}\right)$, the space $\operatorname{Fib}(f) \sim \Omega^{n} M$ when $f$ is nullhomotopic and is empty otherwise.

Proof of 2.4. The inclusion $V \subseteq W$ induces a fibration $p: M^{W} \rightarrow M^{V}$ with $\operatorname{Fib}(f)$ as fiber over $F$. Using adjointness in the usual way, questions about extending map from $K \times V$ to $K \times W$ may be replaced by lifting problems with respect to $p$. Let $M_{a}^{V}$ be the subspace of maps with Lipschitz constant $\leq a$, and let $p_{a}: E \rightarrow M_{a}^{V}$ be the restriction of $p$ over $M_{a}^{V}$.

2.5. Lemma. The map $p_{a}$ satisfies the hypotheses of the Bounded Lifting lemma (2.2 above).

Proof. (a) Using the Arzela-Ascoli theorem, one shows that $M_{a}^{V}$ is compact.

(b) $M^{V}$ is locally contractible [6]. Hence, $p$ is locally fiber-homotopy trivial, and consequently so is any induced fibration.

(c) By the Serre spectral sequence, applied to the restriction of $p$ over individual components of $M^{V}$, each component of $\operatorname{Fib}(f)$ has homology of finite type. By [5], each component of $\operatorname{Fib}(f)$ is a nilpotent space, and since by hypothesis there are only a finite number of components, it must have finite type.

Returning to the proof of 2.4, we fix a nonnegative integer $q$ and $C_{q} \subset E$ be a compact subset as guaranteed by the Bounded Lifting lemma. This inclusion corresponds to a map (evaluation) $e: C_{q} \times W \rightarrow M$ such that $\operatorname{Lip}\left(e \mid\left(C_{q} \times V\right) \leq\right.$ $a)$. We need to adjust $e\left(\operatorname{rel} C_{q} \times V\right)$ so that the adjusted map $\bar{e}: C_{q} \times W \rightarrow$ $M$ will have finite Lipschitz constant. Suppose we found such an $\bar{e}$. Let $F: K \times W M$ be a map such that $\operatorname{Lip}(F \mid(K \times V)) \leq a$ and $\operatorname{dim}(K) \leq q$. Adjoint $F$ to a map $G_{0}: K \rightarrow E$ and deform $G_{0}$ vertically to $G_{1}: K \rightarrow C_{q}$. The map $F_{1}=\bar{e} \circ\left(G_{1} \times \mathrm{Id}\right): K \times W \rightarrow M$ is a suitable map since $\operatorname{Lip}\left(F_{1}\right) \leq \operatorname{Lip}(\bar{e}) \equiv \tilde{b}$.

We now construct $\bar{e}$. Let $\left\{V_{i}\right\}$ be the cover of $M$ guaranteed by 2.3. Let $\kappa$ be the associated constant and let $v=\sup \left\{\operatorname{diam}\left(V_{i}\right)\right\}$. By the compactness of $C_{q} \times W$ there exists a triangulation of $W$ such that for every $x \in C_{q}$ and every simplex $\sigma, e(\{x\} \times \sigma) \subset V_{i}$, for some $i$. We assume that we have triangulated $W$ in this fashion.

Let $W^{0}$ be the vertices of $W$ that are not in $V$. Ordering $W^{0}$, we have $W^{0}=\left\{w_{1}, \ldots, w_{j}, \ldots, w_{k}\right\}$ and we define $\bar{e}$ by induction on $j$. Define $\bar{e}=e$ 
on $C_{q} \times V$. Setting $V \equiv L_{0}$, we define $L_{j}$ to be the subcomplex generated by $L_{j-1}$ and $w_{j}$. Clearly $L_{k}=W$. We define $\bar{e}$ inductively on $L_{j}$ given that we have defined it on $L_{j-1}$. Let $\bar{e}=e$ on $C_{q} \times\left\{w_{j}\right\}$ and suppose $\sigma$ is a simplex of $L_{j-1}$ such that $\sigma * w_{j}$ is in $L_{j}$. For $y \in \sigma$ and $x \in C_{q}$, define

$$
\bar{e}\left(x,\left(t \circ y+(1-t) \circ w_{j}\right)\right)=\lambda\left(\bar{e}(x, y), \bar{e}\left(x, w_{j}\right), t\right),
$$

the geodesic between $\bar{e}(x, y)$ and $\bar{e}\left(x, w_{j}\right)$. Notice that since the $V_{i}$ are convex $e\left(x, \sigma * w_{j}\right) \subset V_{i}$ implies the same for $\bar{e}$ and we can deform $e$ to $\bar{e}$ along geodesics.

We must now verify that $\operatorname{Lip}(\bar{e})<\infty$. This amounts to an exercise in local Riemannian geometry. First, because Lip is local in nature it suffices to show $\operatorname{Lip}(\bar{e})$ is bounded on $\sigma * w_{j}$. Let $y_{0}$ and $z_{0}$ be two points of this simplex. Using barycentric coordinates, we write

$$
y_{0}=s y+(1-s) w_{j} \quad \text { and } \quad z_{0}=t z+(1-t) w_{j} .
$$

Suppose $t \geq s$. Defining $z_{1}=s z+(1-s) w_{j}$ and letting $d$ denote the barycentric metric on $W$, we have

$$
d\left(y_{0}, z_{0}\right)^{2}=d\left(y_{0}, z_{1}\right)^{2}+d\left(z_{1}, z_{0}\right)^{2}-2 d\left(y_{0}, z_{1}\right) d\left(z_{1}, z_{0}\right) \cos (\eta),
$$

where $\eta$ is the angle $y_{0} z_{1} z_{0}$. Since $\eta \geq \pi / 4$, we have $\cos (\eta) \leq 1 / \sqrt{2}$. Hence

$$
\begin{aligned}
d\left(y_{0}, z_{0}\right)^{2} & \geq d\left(y_{0}, z_{1}\right)^{2}+d\left(z_{1}, z_{0}\right)^{2}-\sqrt{2} d\left(y_{0}, z_{1}\right) d\left(z_{1}, z_{0}\right), \\
& =d\left(y_{0}, z_{1}\right)^{2} / 2+\left(d\left(y_{0}, z_{1}\right) / \sqrt{2}-d\left(z_{1}, z_{0}\right)\right)^{2},
\end{aligned}
$$

so

$$
d\left(y_{0}, z_{0}\right) \geq d\left(y_{0}, z_{1}\right) / \sqrt{2} \text { and } d\left(y_{0}, z_{0}\right) \geq d\left(z_{1}, z_{0}\right) / \sqrt{2} \text {. }
$$

Let $Y_{0}, Z_{0}, Z_{1}$, etc. be the images of $y_{0}, z_{0}, z_{1}$, etc. under $\bar{e}$. Using the symbol $d$ ambiguously we have

$$
\frac{d\left(Y_{0}, Z_{0}\right)}{d\left(y_{0}, z_{0}\right)} \leq \frac{d\left(Y_{0}, Z_{1}\right)}{d\left(y_{0}, z_{0}\right)}+\frac{d\left(Z_{1}, Z_{0}\right)}{d\left(y_{0}, z_{0}\right)} \leq \sqrt{2}\left(\frac{d\left(Y_{0}, Z_{1}\right)}{d\left(y_{0}, z_{1}\right)}+\frac{d\left(Z_{1}, Z_{0}\right)}{d\left(z_{1}, z_{0}\right)}\right) .
$$

But

$$
\frac{d\left(Y_{0}, Z_{1}\right)}{d\left(y_{0}, z_{1}\right)}=\frac{d\left(Y, W_{j}\right)}{d\left(y, w_{j}\right)}
$$

since $Y, Y_{0}, Z_{1}$, and $W_{j}$ lie along the same geodesic. This second term is bounded since $\sigma$ is compact. Moreover, $d\left(z_{1}, z_{0}\right)=s d(y, z)$ and $d\left(Z_{1}, Z_{0}\right) \leq$ $\kappa s d(Y, Z)$, where $\kappa$ is as defined in 2.3. Thus

$$
\frac{d\left(Z_{1}, Z_{0}\right)}{d\left(z_{1}, z_{0}\right)} \leq \kappa \frac{d(Y, Z)}{d(y, z)}=\kappa \lambda_{j-1},
$$

where $\lambda_{j-1}$ is the Lipschitz bound on $L_{j-1}$.

2.6. Final remarks. Theorem $\mathrm{A}$ is, of course, not the only obvious application of 2.4. Let $M$ be an $n$-sphere or a manifold covered by an $n$-sphere. If the pair $(W, V)$ is sufficiently connected, then the hypothesis of 2.4 is satisfied because the homotopy groups of $M$ will be finite in the appropriate dimensions. 


\section{REFERENCES}

1. A. Calder and J. Siegel, On the width of homotopies, Topology 19 (1980), 209-220.

2. __ Homotopies of bounded width are almost Lipschitz, Topology Appl. 14 (1982), 117-129.

3. A. Calder, J. Siegel, and F. Williams, The width of homotopies into spheres, Topology 21 (1982), 281-290.

4. M. Gromov, Homotopical effects of dilation, J. Differential Geom. 13 (1978), 303-310.

5. P. Hilton, G. Mislin, and J. Roitberg, Localization of nilpotent groups and spaces, NorthHolland Math. Stud., no. 15, Amsterdam, 1975.

6. A. Lundell and S. Weingram, The topology of $C W$-complexes, Van Nostrand Reinhold, New York, 1969.

7. R. Olivier, Über die Dehnung von Spharenabbildungen, Invent. Math. 1 (1966), 380-390.

8. J. Roitberg, Dilatation phenomena in the homotopy groups of spheres, Adv. in Math. 15 (1975), 198-206.

9. J. Siegel, and F. Williams, Numerical invariants of homotopies into spheres, Pacific J. Math. 110 (1984), 417-428.

10. __ Variational invariants of Riemannian manifolds, Trans. Amer. Math. Soc. 294 (1984), 417-428.

11. __ Uniform bounds for equivariant homotopies, Topology Appl. (to appear).

Department of Mathematics, University of Missouri-St. Louis, St. Louis, Missouri 63121

Department of Mathematics, New Mexico State University, Las Cruces, New Mexico 88003 